\title{
Teorías implícitas respecto a la enseñanza y el aprendizaje: ¿Existen diferencias entre profesores en ejercicio y estudiantes de pedagogía?
}

\author{
Implicit theories about teaching and learning: Are there differences between \\ in-service and pre-service teachers?
}
Teorias implícitas sobre ensino-aprendizagem: há diferenças entre professores em exer- cício e estudantes de Pedagogia?

\section{Viviana Gómez', Paula Guerra}

${ }^{1}$ Pontificia Universidad Católica de Chile, Facultad de Educación, vgomezn@uc.cl, 56-2-3547988

${ }^{2}$ Pontificia Universidad Católica de Chile, pfguerra@uc.cl, 56-2-3545311.

\begin{abstract}
RESUMEN
Esta investigación indaga los perfiles teóricos sobre el aprendizaje y enseñanza que sostienen 837 profesores en servicio y estudiantes de pedagogía, a través de un cuestionario de dilemas. Ambos grupos sostienen enfoques teóricos interpretativos. Los profesores en servicio se diferencian significativamente sólo respecto a experiencia profesional, siendo los novatos más constructivistas que los más experimentados. Los estudiantes de pedagogía presentan diferencias significativas por universidad de origen y rendimiento y no se diferencian de los profesores novatos. Los estudiantes son más constructivistas que los profesores respecto a cómo enseñar conceptos y procedimientos. Los profesores son más constructivistas respecto a la motivación, evaluación y enseñanza de actitudes. Se discuten las implicaciones para la formación inicial y continua de profesores.
\end{abstract}

Palabras clave: constructivismo, formación docente, teorías implícitas sobre aprendizaje y enseñanza, estudiantes de pedagogía.

\begin{abstract}
This study explores the theoretical profiles in the teaching and learning process of 837-service and pre-service teachers through a dilemma's questionnaire. Both teachers and students have mainly interpretative approaches. In-service teachers have significant differences only on professional experience, where novice teachers are more constructivists than experienced teachers. Pre-service teachers have significant differences comparing university and academic performance, and student teachers have no differences in comparison with novice teachers. Pedagogy student are more constructivist than teachers in relation to the conceptual and procedural teaching, but teachers are more constructivist in relation to motivation, assessment, and attitudes' teaching. Implications for initial teacher's education and professional development programs are discussed.

Key words: constructivism, teacher education, teaching, learning, teaching's student.
\end{abstract}

\section{RESUMO}

Por meio de questionário sobre seus dilemas, esta investigação questiona os perfis teóricos a respeito do processo ensino-aprendizagem defendidos por 837 docentes em exercício e estudantes de Pedagogia. Ambos sustentam enfoques teóricos interpretativos. Os professores em exercício diferenciam, significativamente, apenas em relação à experiência profissional, sendo os professores iniciantes mais construtivistas do que aqueles com maior tempo na profissão. Os estudantes de Pedagogia apresentam diferenças significativas se considerado o local de formação, o rendimento acadêmico e se igualam aos professores novatos. Os estudantes são mais construtivistas que os professores no que diz respeito a como ensinar conceitos e procedimentos. Os professores são mais construtivistas no que diz respeito à motivação, à avaliação e ao ensino de atitudes. Discutem-se as implicações para a formação inicial e continuada de professores.

Palavras chave: construtivismo, formação docente, ensino, aprendizagem. 


\section{INTRODUCCIÓN}

Para dar respuesta a los desafíos que impone la sociedad contemporánea a la educación, en nuestro país se promulgó una reforma educacional que se orienta a nuevas metas de aprendizaje y hacia una nueva comprensión de la enseñanza claramente basadas en una visión constructivista. Buscando alcanzar estas metas, ha intentado mejorar tanto las condiciones materiales como humanas de la actual oferta educativa a través de una importante inversión de fondos públicos orientada a la preparación del profesorado a través de distintas modalidades de desarrollo profesional docente. Sin embargo, los cambios aún son bastante desalentadores. La literatura actual señala que los profesores en servicio no comprenden todavía qué implica llevar a la práctica una enseñanza basada en el enfoque constructivista (Prawat, 1992; Windschitl, 2002) y que, a lo más, muestran una enseñanza "en transición" respecto al espíritu de la reforma (Beca et al., 2003; Cox, 2003). Junto con esto, los análisis de los programas de formación inicial de profesores tampoco parecen estar entregando al sistema educativo profesores que realicen una práctica de enseñanza basada en el constructivismo (Ávalos, 2004). ¿Por qué ha sido tan difícil llevar a los profesores a una enseñanza orientada hacia el constructivismo? La investigación señala que el núcleo duro de la formación orientada al constructivismo se encuentra en cómo éste se modela y se lleva a la práctica durante la formación (Korthagen, Loughran \& Russell, 2006; Lunenberg, Korthagen \& Swennen, 2007), no obstante, como señala Windschitl (2002), su puesta en marcha depende no sólo de variables conceptuales y pedagógicas, sino también de variables culturales y políticas. El estado ha hecho además importantes esfuerzos por estimular la innovación en los programas de formación inicial de profesores, al inicio de los años 2000, tales como el proyecto PFFID (Programa de Fortalecimiento de la Formación Inicial Docente) y los actuales proyectos MECESUP (Programa de Mejoramiento de la Calidad de la Educación Superior). Se podría esperar, entonces, que a fines de la primera década del Siglo XXI debieran observarse resultados positivos en las teorías del aprendizaje y la enseñanza que sostienen los nuevos profesores que egresan de nuestras universidades. Pero, a pesar del convencimiento de que es la forma en que ocurre el aprendizaje, estamos lejos de saber cómo se modela o si es posible impactar las concepciones previas en forma radical o sólo se logra una yuxtaposición o una compartimentación de las teorías del aprendizaje (Rodrigo y Correa, 2001). Comprendiendo que el cambio hacia un enfoque constructivista implica numerosos desafíos para los programas de formación inicial y continua de profesores, nuestro estudio tiene por objetivo conocer los perfiles teóricos sobre el aprendizaje y la enseñanza que sostienen tanto los profesores en servicio como los estudiantes de pedagogía y observar si estos últimos poseen un perfil teórico más acorde con las actuales demandas de reforma educacional.

\section{MARCO CONCEPTUAL}

La literatura de los años 90 mostraba que los futuros profesores ingresan a los programas de formación con cuerpos de conocimientos sobre la pedagogía muy bien consolidados y que no cambian a pesar de su paso por una institución formal de enseñanza (Woolfolk, Davis \& Pape, 2006). Una posible explicación se encontraría a la base misma de la visión constructivista. Los sujetos son constructores activos de significado 
y lo elaboran tanto a partir de la experiencia previa como de la negociación con otros, eligiendo consciente o inconscientemente, entre valores y hábitos dentro del ambiente social (von Wright, 1997). Esta elección es importante para la comprensión, ya que es la base de lo que consideran relevante en diversas situaciones y dirige la atención hacia ellas (Dweck, 1996). Este conocimiento previo se basa tanto en sus experiencias formales como informales en la vida diaria, las que permiten el desarrollo de un conjunto coherente de ideas, pre-concepciones y creencias conocido principalmente con el nombre de teorías implícitas (Rodrigo, Rodríguez \& Marrero 1993), considerados como conocimiento ingenuo (Hatano \& Inagaki, 1994; Keil, 1992). Se caracteriza por ser durable, funcional, fácilmente recuperable y, lo que dificulta la posibilidad de modificación, es que tiene un alto poder explicativo para las situaciones cotidianas del aula. De esta forma, el conocimiento ingenuo a menudo compite con el conocimiento nuevo especialmente si este último no es consistente con el anterior, puesto que tanto los futuros profesores y los que están en servicio, han pasado más de 12.000 horas en la escuela durante su propia escolarización, desde donde han inferido modelos implícitos acerca de lo que es la enseñanza y el aprendizaje (Strauss y Shilony, 1994). El presente estudio se inscribe en el campo de las teorías implícitas sobre la enseñanza y el aprendizaje. Las que corresponden a enfoques representacionales sobre la naturaleza implícita y explícita del conocimiento, caracterizado por representaciones complejas que funcionan como un conjunto de principios que restringen la forma de afrontar, interpretar o atender a las situaciones de enseñanzaaprendizaje y que a su vez le dan cohesión y organización (Pozo, Scheuer, Mateos \& Pérez Echeverría 2006). El conocimiento previo, las pre-concepciones y las creencias que llevan los futuros profesores a los programas de formación han sido descritos y caracterizados por varios investigadores (Wideen, Mayer-Smith \& Moon 1998; Wolfolk et al., 2006). Estos investigadores indican que los estudiantes de pedagogía presentan una serie de teorías implícitas las que se pueden caracterizar como: una excesiva confianza en los componentes afectivos sin considerar los aspectos cognitivos del proceso de enseñanzaaprendizaje; una tendencia a explicar las situaciones basándose primordialmente en el recuerdo de incidentes específicos; la creencia de que algunas situaciones están más allá del control del profesor y que, además, no están sujetas a cambio; la consideración de que la enseñanza es la transmisión de cuerpos intactos de conocimiento desde el conocedor al aprendiz, etc. Como se puede constatar, estas ideas son contradictorias con lo que plantea la aproximación constructivista de la enseñanza y el aprendizaje (Brooks \& Brooks, 1993; Brown, 1994).

\subsection{TEORÍAS IMPLÍCITAS DE LOS PROFESORES Y ESTUDIANTES}

En España, Pozo et al. (2006) han descrito las teorías implícitas sobre la enseñanza y el aprendizaje de los profesores en servicio, luego de haber realizado algunos estudios con profesores de enseñanza primaria y secundaria. Esta descripción nos permite comprender cómo la teoría construida por el profesor a partir de su experiencia anterior, le permite explicar e interpretar dilemas del aula, y cómo estos enfoques se acercan en mayor o menor medida a algunas aproximaciones teóricas formales hacia el aprendizaje como son el conductismo, el procesamiento de la información y el propio constructivismo. Para estos autores estas teorías espontáneas son básicamente tres: la teoría directa, teoría interpretativa y teoría constructiva. La teoría directa, con el supuesto epistemológico de que 
el conocimiento es una copia fiel de la realidad, reduce el aprendizaje a la reproducción de estímulos o conductas sin la mediación de procesos psicológicos superiores. Asume una concepción dualista del conocimiento, concibiendo tanto a sujeto como objeto como entidades separadas, un objeto que existe independientemente del sujeto y que puede ser estudiado objetivamente (sin sesgos) por un sujeto externo a él. Según Pozo et al. (2006), es afín a una visión conductista del aprendizaje. La teoría interpretativa conserva el supuesto epistemológico del enfoque anterior, pero el aprendizaje es el resultado de la actividad personal del sujeto, quien opera con una serie de procesos psicológicos mediadores. Esta teoría es cercana a los modelos de procesamiento de información el que, si bien asume un aprendizaje activo, es igualmente reproductivo (Pozo et al., 2006). La teoría constructivista admite la existencia de saberes múltiples, el perspectivismo y relativismo. Todo conocimiento es una construcción contextualizada y, por tanto, relativa y con diferentes grados de certidumbre. El aprendizaje implica procesos mentales reconstructivos de las propias representaciones anteriores en diálogo con el nuevo contenido. El aprendiz tiene conciencia de las condiciones en las que ocurre el aprendizaje, el que implica autorregulación de la propia actividad y un ajuste de los procesos metacognitivos que regulan su aprendizaje (Pozo et al., 2006). Considerando estos planteamientos, nuestro estudio ha querido responder a las siguientes preguntas ¿Existe coherencia en el enfoque teórico que utilizan tanto los estudiantes de pedagogía como los profesores en servicio para interpretar los procesos de enseñanza y aprendizaje que ocurren en la escuela? ¿Difieren los futuros profesores de aquellos que están en servicio respecto las teorías implícitas sobre la enseñanza y el aprendizaje que sostienen? ¿Se mantiene la tendencia mostrada por los estudios de los años 90 que señalan la falta de impacto de los programas de formación, en la transformación de la visión realista con la que ingresan a la universidad? ¿Cuáles son las dimensiones de la enseñanza y el aprendizaje en que aparecen las mayores semejanzas y diferencias entre ambos grupos?

\section{METODOLOGÍA}

\subsection{MUESTRA}

Se utilizó una muestra representativa de 409 estudiantes que cursaban su último año de Pedagogía -con un criterio de confiabilidad de 95\%- de un universo de 3.890 estudiantes de Pedagogía en Educación General Básica y de 13.941 en Pedagogía en Educación Media de 17 Universidades pertenecientes al Consejo de Rectores del país. La muestra contenía un número proporcional de estudiantes de acuerdo al porcentaje por Universidad. Se seleccionó, también, una muestra representativa de 428 profesores de la Región Metropolitana, con un criterio de confiabilidad de 95\%, de un total de 37.797 profesores en ejercicio, distribuida proporcionalmente de acuerdo a dependencia administrativa, nivel escolar y género de los profesores. 


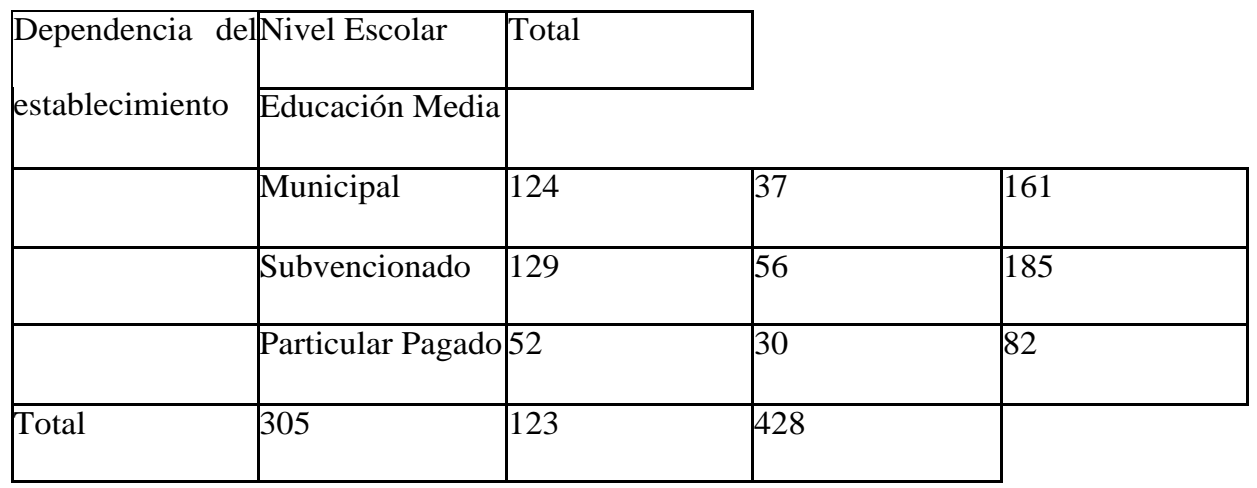

Tabla 1. Distribución de la muestra de Profesores en Servicio Región Metropolitana

Todos los participantes firmaron un consentimiento informado, aceptado su participación en el estudio. La Tabla 1 muestra la distribución de la muestra de profesores y la Tabla 2 la de estudiantes de pedagogía.

\begin{tabular}{|l|c|c|c|l|c|c|c|}
\hline Universidad & Nivel & Universidad & Nive & \multicolumn{2}{l}{} \\
\hline Básica & Media & Total & Básica & Media & \multicolumn{1}{l}{ Total } & \multicolumn{2}{l}{} \\
\hline Univ. 1 & 6 & 6 & 12 & Univ. 10 & 0 & 16 & 16 \\
\hline Univ. 2 & 25 & 31 & 56 & Univ. 11 & 0 & 6 & 6 \\
\hline Univ. 3 & 2 & 13 & 15 & Univ. 12 & 16 & 18 & 34 \\
\hline Univ. 4 & 5 & 9 & 14 & Univ. 13 & 4 & 8 & 12 \\
\hline Univ. 5 & 8 & 2 & 10 & Univ. 14 & 16 & 0 & 16 \\
\hline Univ. 6 & 10 & 40 & 50 & Univ. 15 & 10 & 62 & 72 \\
\hline Univ. 7 & 7 & 0 & 7 & Univ. 16 & 1 & 30 & 31 \\
\hline Univ. 8 & 6 & 12 & 18 & Univ. 17 & 4 & 4 & 8 \\
\hline Univ. 9 & 11 & 21 & 32 & Total & 131 & 278 & 409 \\
\hline
\end{tabular}

Tabla 2. Distribución de la muestra de Estudiantes de Pedagogía a nivel nacional 
El promedio de edad de los profesores en ejercicio era de 40 años y su experiencia docente de 15 . Además, un $35.3 \%$ de éstos contaba con postítulo. El promedio de edad de los estudiantes de pedagogía era de 23 años y, en su mayoría, se encontraban en el proceso de práctica profesional realizada en el quinto año de carrera. Respecto a la distribución por género, en el caso de los profesores en ejercicio, un $69.6 \%$ eran mujeres, mientras que un $30.4 \%$ eran hombres. Un $64.3 \%$ de los estudiantes de pedagogía eran mujeres y un $35.2 \%$ eran hombres.

\subsection{INSTRUMENTO:}

Se realizó una adaptación del cuestionario elaborado por Martín et al. (2001, citado en Pozo et al., 2006) mediante un proceso de revisión del lenguaje y validación jueces. El cuestionario consta de 19 dilemas referidos a situaciones cotidianas de la vida escolar. Cada dilema tiene tres posibilidades de respuesta, representando cada alternativa un enfoque teórico distinto: el enfoque directo, interpretativo o constructivista, para interpretar dicho fenómeno. Los dilemas se refieren a 6 dominios específicos sobre los cuales se aplican estas teorías. Éstos son: la enseñanza de conceptos, procedimientos y actitudes, la motivación, la evaluación y la relación entre capacidades y contenidos. Un ejemplo de dilema de este cuestionario, es el siguiente:

15. En una discusión acerca de cómo hacer preguntas para evaluar los distintos grados de aprendizaje, los profesores exponen distintos puntos de vista: (escoge la opción que más se acerque a tu opinión personal).

a) Las preguntas deben ser suficientemente amplias para que puedan contestarse en diferentes grados de profundidad, aunque la evaluación pierda objetividad.

b) Las preguntas deben ser las mismas y lo más objetivas posible para asegurar que los aprendizajes del curso se han logrado

c) si se ha trabajado en el aula atendiendo a los distintos niveles de aprendizaje, la evaluación ya no necesita diferenciarse y puede ser igual para todos.

El análisis psicométrico del cuestionario arrojó una confiabilidad moderada (Alpha de Cronbach 0.74).

\subsection{ANÁLISIS DE DATOS}

Los resultados del cuestionario de dilemas hacen difícil, sino cuestionable, la posibilidad de sumar las puntuaciones y establecer conclusiones en relación a la puntuación total, debido a que no existe una continuidad entre los tres enfoques teóricos. La forma en que se distribuían las elecciones de los participantes en el estudio, mostraba una proporción de respuestas correspondientes a los tres enfoques y no enfoques puros. Por esta razón realizamos un Standard setting para determinar criterios útiles para reconocer el perfil teórico de cada sujeto, realizando los siguientes pasos: revisamos la literatura para determinar las competencias y los enfoques posibles de utilizar para interpretar diferentes aspectos de la enseñanza y del aprendizaje; luego discutimos los perfiles y determinamos 
los criterios de corte y los aplicamos a una muestra de los sujetos. A continuación, se analizó el funcionamiento de los criterios y se discutieron los casos complejos, haciendo los ajustes que permitieron determinar los perfiles teóricos de toda la muestra. Así se llegó a los criterios que se presentan en la Tabla 3 .

\begin{tabular}{|l|l|l|l|}
\hline Perfil teórico & Directa & $\begin{array}{l}\text { Interpretativa } \\
\text { (procesamiento de Ia }\end{array}$ & \\
& (conductismo) & Constructivista \\
\hline información) & \\
\hline Interpretativ0+ & $\leq 4(<20 \%)$ & $5(<21 \%)$ & $<10(<53 \%)$ \\
\hline Constructivista- & $0-2(<10 \%)$ & $5-7(>30 \%)$ & $12-14(60-74 \%)$ \\
\hline Constructivista+ & $0-1(<0,5 \%)$ & $0-3(<35 \%)$ & $>15(>75 \%)$ \\
\hline
\end{tabular}

Tabla 3. Criterios para determinar el perfil teórico predominante en los sujetos considerando el $\mathrm{n}^{\mathrm{o}} \mathrm{y}$ porcentaje de respuestas correspondiente a cada tipo de teoría.

Finalmente se entrenó a dos estudiantes de doctorado para aplicar los criterios de corte establecidos a la totalidad de la muestra. De acuerdo a estos criterios de corte, se determinaron los perfiles interpretativo-, interpretativo+, constructivista- y constructivista+, en función de los cuales se efectuaron los análisis. Posteriormente, se realizaron análisis descriptivos y análisis comparativos utilizando la prueba de Chi-cuadrado. Además, se estimó el tamaño del efecto (d), cuando la prueba arrojaba diferencias significativas. Los análisis se efectuaron con los programas SPSS versión 12.0 y GPower 3.0.

\section{RESULTADOS}

Presentaremos los resultados del grupo de profesores, luego el de los estudiantes de pedagogía y, finalmente, la comparación entre estudiantes y profesores en servicio.

\subsection{RESULTADOS PROFESORES EN EJERCICIO}

En los datos descriptivos de la Tabla 4 vemos que el perfil teórico predominante es el Interpretativo+, es decir el $43.7 \%$ de los profesores encuestados tiende a interpretar situaciones educativas respondiendo a poco más de la mitad de los dilemas con un enfoque constructivista pero, al mismo tiempo, utiliza proporciones más o menos semejantes de interpretaciones ligadas a la teoría directa y al procesamiento de la información. Un $29 \%$ de los profesores presenta un perfil Interpretativo- en el que se observa un mayor 
porcentaje de respuestas de la teoría directa, lo que se traduciría en una visión reproductiva del aprendizaje. Un 22.4\% presenta un perfil Constructivista-, que indicaría una visión predominantemente constructivista, pero que aún se ve matizada con algunas nociones ligadas al procesamiento de la información, teoría que se considera epistemológicamente distinta al constructivismo. Por último, sólo un $4.9 \%$ de los profesores encuestados presenta una visión puramente constructivista.

\begin{tabular}{|c|c|c|c|c|c|c|c|c|}
\hline $\begin{array}{l}\text { Perfil } \\
\text { teórico/Dependencia }\end{array}$ & Municipa & $\begin{array}{l}\text { Particular } \\
\text { subvencionado }\end{array}$ & $\begin{array}{l}\text { Particular } \\
\text { pagado }\end{array}$ & Total & & & & \\
\hline $\mathrm{N}$ & $\%$ & $N$ & $\%$ & $\mathrm{~N}$ & $\%$ & $\mathrm{~N}$ & $\%$ & \\
\hline Interpretativo- & 57 & 35.4 & 51 & 27.6 & 16 & 19.5 & 124 & 29 \\
\hline Interpretativo+ & 60 & 37.3 & 90 & 48.6 & 37 & 45.1 & 187 & 43.7 \\
\hline Constructivista & 36 & 22.4 & 38 & 20.5 & 22 & 26.8 & 96 & 22.4 \\
\hline Constructivistat & 8 & 5 & 6 & 3.2 & 7 & 8.5 & 21 & 4.9 \\
\hline Total & 161 & & 185 & & 82 & & 428 & \\
\hline
\end{tabular}

Tabla 4. Estadísticos descriptivos de la muestra total de profesores en servicio por tipo de dependencia.

Al comparar a los profesores por dependencia administrativa de los colegios, el perfil teórico predominante es el Interpretativo+, presente en el $45.1 \%$ de los profesores de colegios particulares, el $48.6 \%$ de subvencionados y el 37.3 de municipales. El segundo enfoque predominante muestra que los profesores de los colegios municipales tienen más tendencia hacia el enfoque interpretativo- (35.4\%), mientras que los particulares tienen más tendencia hacia el enfoque constructivista- (26.8\%). Los tres grupos presentan poca frecuencia del enfoque constructivista+, pero los profesores de colegios subvencionados tienen la más baja presencia y los profesores de colegios particulares, la más alta. Estadísticamente, sin embargo, estas diferencias no son significativas.

Diferencias por años de experiencia. Los datos referidos a los años de experiencia profesional de los docentes de la Tabla 5 muestra que, a medida que aumenta la experiencia profesional, encontramos mayor proporción de profesores con enfoque interpretativo-, llegando a un $43.4 \%$ entre los profesores con más de 22 años en la profesión. Por otra parte, los profesores más jóvenes, con hasta 6 años de antigüedad, presentan la más alta proporción del enfoque constructivista+, aunque no alcanza el 10\% del total de profesores. 


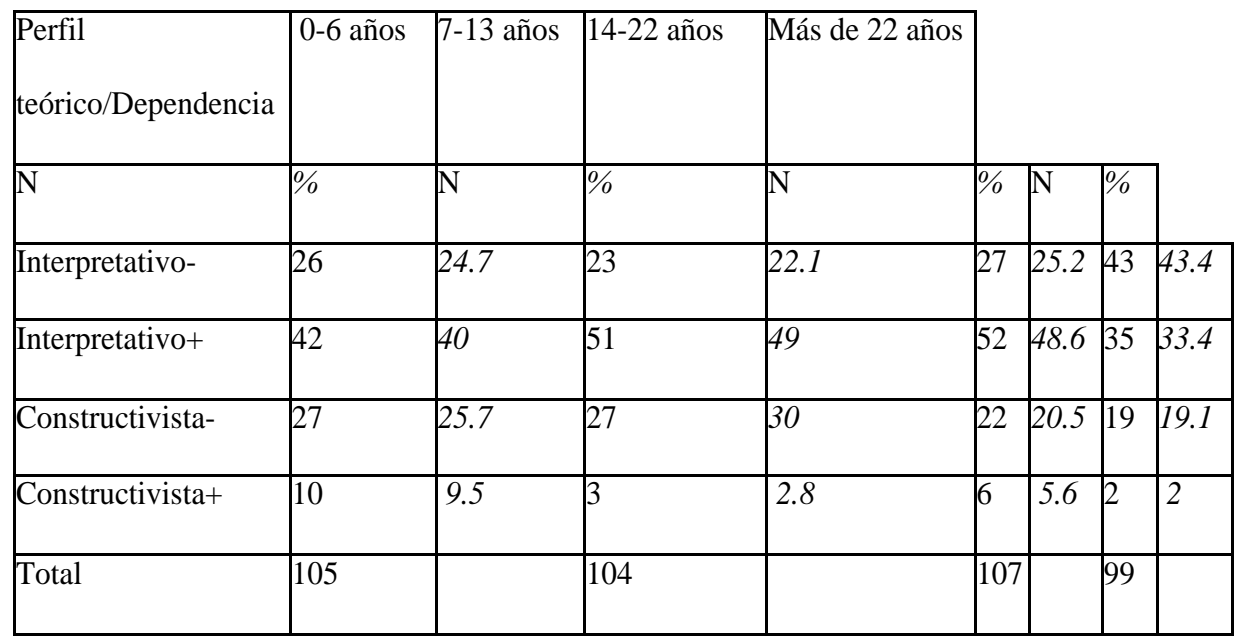

Tabla 5. Estadísticos descriptivos acerca de los años de experiencia docente de los profesores

El análisis estadístico muestra que existen diferencias estadísticamente significativas $(\mathrm{X} 2(9, \mathrm{~N}=415)=21.888, \mathrm{p}<.009)$ según experiencia profesional, a favor de los profesores más jóvenes quienes tienen mayor porcentaje de sujetos con perfiles Constructivista- y Constructivista+ comparados con los otros grupos. Sin embargo, el d es de 0.23 , lo que se considera un valor medio. Al compararlos por dependencia administrativa de la escuela, sub-sector o disciplina, ciclo de enseñanza, desarrollo profesional o tipo de institución en la que se formaron, no se encuentran diferencias significativas entre los profesores.

\subsection{RESULTADOS ESTUDIANTES DE PEDAGOGÍA}

Los estadísticos descriptivos de la tabla 6 indican que, al igual que en el grupo de profesores, el mayor porcentaje de estudiantes de pedagogía (un 38.9\%) muestra un perfil Interpretativo+, es decir, que tienden a interpretar situaciones educativas con poco más de la mitad de respuestas constructivistas pero, en proporciones más o menos semejantes, se orientan también a interpretaciones ligadas al enfoque directo y del procesamiento de la información. Un $27 \%$ de los estudiantes presentan un perfil Interpretativo-, es decir, presentando la mitad de las respuestas de tipo constructivista pero ligadas a un alto porcentaje de respuestas relacionadas con el realismo, en la que el porcentaje de respuestas de tipo directa ligada al conductismo es mayor que el de respuestas ligadas al procesamiento de la información. Un $22.1 \%$ de los estudiantes se ubica en un perfil Constructivista-, lo que indica una visión del aprendizaje predominantemente constructivista, pero que aún se ve mezclada con algunas nociones ligadas a los otros enfoques. Por último, sólo un $11.7 \%$ de los estudiantes encuestados presenta una visión del aprendizaje puramente constructivista+. 


\begin{tabular}{|l|l|l|}
\hline Perfil teórico/Dependencia & $\mathrm{N}$ & $\%$ \\
\hline Interpretativo- & 110 & 26.9 \\
\hline Interpretativo+ & 160 & 39.1 \\
\hline Constructivista- & 91 & 22.2 \\
\hline Constructivista+ & 48 & 11.7 \\
\hline Total & 409 & 100 \\
\hline
\end{tabular}

Tabla 6. Estadísticos descriptivos de teoría predominante en la muestra de estudiantes de pedagogía nacionales

Diferencias por Institución de Formación. Al comparar los resultados que obtienen los estudiantes de pedagogía de acuerdo a la institución formadora, se encuentran diferencias significativas $(\mathrm{X} 2(48, \mathrm{~N}=409)=127.267, \mathrm{p}<.00)$. Esto implica que los distintos programas de formación en marcha en el país impactarían diferencialmente el perfil teórico que desarrollan sus estudiantes, formando algunas instituciones a futuros profesores eminentemente constructivistas en su enfoque sobre la enseñanza y el aprendizaje, mientras que otras parecen más bien consolidar los modelos transmisivos. El d fue de 0.32 , el que se considera un valor medio. Por otra parte, si revisamos los datos de la tabla 7, considerando aquellas instituciones que han desarrollado innovaciones (adjudicándose proyectos PFFID), se aprecia que no existen diferencias significativas entre los estudiantes de las instituciones que innovaron en la formación y aquellas que no lo hicieron.

\begin{tabular}{|l|l|l|l|l|}
\hline Perfil & Con & Sin & \multicolumn{2}{l|}{} \\
teórico/Dependencia & Innovación & Innovación & \multicolumn{2}{l|}{} \\
\hline Interpretativo- & 61 & 27.5 & 49 & 26.2 \\
\hline Interpretativo+ & 94 & 42.3 & 66 & 35.3 \\
\hline Constructivista & 44 & 19.8 & 47 & 25.1 \\
\hline Constructivista+ & 23 & 10.4 & 25 & 13.4 \\
\hline Total & 222 & & 187 & \\
\hline
\end{tabular}

Tabla 7. Distribución de estudiantes según participación en proyecto PFFID 
Diferecias por rendimiento académico. La Tabla 8 nos muestra la distribución de los estudiantes de acuerdo al promedio de notas acumulado en su formación pedagógica. Como podemos observar, los perfiles teóricos se mantienen más o menos estables cuando los estudiantes presentan un rendimiento por debajo del promedio 6.0 (sobresaliente en otros países). Sólo aquellos estudiantes con excelentes resultados académicos muestran mayor porcentaje del enfoque constructivista+, sin embargo, éstos superan levemente el $10 \%$ del grupo total.

\begin{tabular}{|c|c|c|c|c|c|}
\hline $\begin{array}{l}\text { Promedio } \\
\text { acumulado }\end{array}$ & Perfil teórico & Total & & & \\
\hline Interpretativa- & Interpretativa+ & C onstructivista- & Constructivista+ & & \\
\hline $\bar{N}$ & $\%$ & & & & \\
\hline 4,1 a 4,5 & 4 & 4 & 2 & 0 & 102.4 \\
\hline 4,6 a 5,0 & 25 & 35 & 20 & 3 & 8320 \\
\hline 5,1 a 5,5 & 50 & 61 & 30 & 17 & $\begin{array}{l}158 \\
39\end{array}$ \\
\hline 5,6 a 6,0 & 26 & 43 & 26 & 13 & $\begin{array}{l}108 \\
27\end{array}$ \\
\hline 6,1 a 6,5 & 5 & 13 & 12 & 13 & 4311 \\
\hline 6,6 a 7,0 & 0 & 0 & 0 & 2 & 20.4 \\
\hline Total & 110 & 156 & 90 & 48 & $\begin{array}{l}404 \\
100\end{array}$ \\
\hline
\end{tabular}

Tabla 8. Distribución del perfil teórico de los estudiantes de pedagogía de último año, de acuerdo al rendimiento (promedio de notas acumulado).

La prueba Chi-cuadrado revela que existen diferencias significativas entre los estudiantes de acuerdo al promedio de notas acumulado en sus carreras (X2 (15, N=404) $=42.160, \mathrm{p}<.000$ ), pero con un d de 0.26 , considerado un valor medio. No se evidencian diferencias estadísticamente significativas al compararlos por ciclo de enseñanza ni por género. 


\subsection{RESULTADOS COMPARATIVOS PROFESORES EN EJERCICIO Y ESTUDIANTES DE PEDAGOGÍA}

Los datos de la Tabla 9 muestran que en ambos grupos predomina el enfoque interpretativo+, lo que implicaría que estos sujetos tienen casi igual probabilidad de utilizar enfoques teóricos que son epistemológicamente contradictorios para interpretar los procesos de enseñanza y aprendizaje. Por otra parte, es importante señalar que aunque ambos grupos se distribuyen en forma más o menos similar, se observa mayor porcentaje de respuestas constructivistas entre los estudiantes. La prueba Chi cuadrado muestra que existen diferencias estadísticamente significativas entre ambos grupos $(X 2(3, N=837)$ $=13.213, \mathrm{p}<.004)$. No obstante, el d es de 0.14 , considerado un valor bajo, por lo que podríamos considerarlo como un resultado más bien débil.

\begin{tabular}{|l|l|l|l|l|}
\hline Perfil & Profesores & Estudiantes & \multicolumn{2}{l}{} \\
teórico/Dependencia & & & & \\
\hline$N$ & $\%$ & $N$ & 110 & 26.9 \\
\hline Interpretativo- & 124 & 29 & 160 & 39.1 \\
\hline Interpretativo+ & 187 & 43.7 & 91 & 22.2 \\
\hline Constructivista- & 96 & 22.4 & 48 & 11.7 \\
\hline Constructivista+ & 21 & 4.9 & 428 & \\
\hline Total & 409 & & & \\
\hline
\end{tabular}

Tabla 9. Frecuencias Teorías Estudiantes-Profesores

\subsection{OTROS ANÁLISIS}

\subsubsection{Comparación estudiantes de pedagogía y profesores novatos}

Dado que los análisis muestran que estudiantes de último año de pedagogía se diferencian de los profesores en ejercicio y que, dentro de este último grupo, existen diferencias de acuerdo a los años de experiencia, nos interesó saber si habría diferencia entre los estudiantes de pedagogía y el grupo de profesores más jóvenes. Los datos muestran que no se encuentran diferencias significativas entre ambos grupos.

\subsubsection{Diferencias en las dimensiones de la enseñanza y el aprendizaje evaluadas, entre estudiantes de pedagogía y profesores en servicio}

Aunque era difícil distinguir una tendencia en algunas de las dimensiones debido al pequeño número de ítemes por dimensión, decidimos intentar la determinación del enfoque teórico para cada una de las 6 dimensiones del instrumento considerando la categorización que se observa en la Tabla 10. 


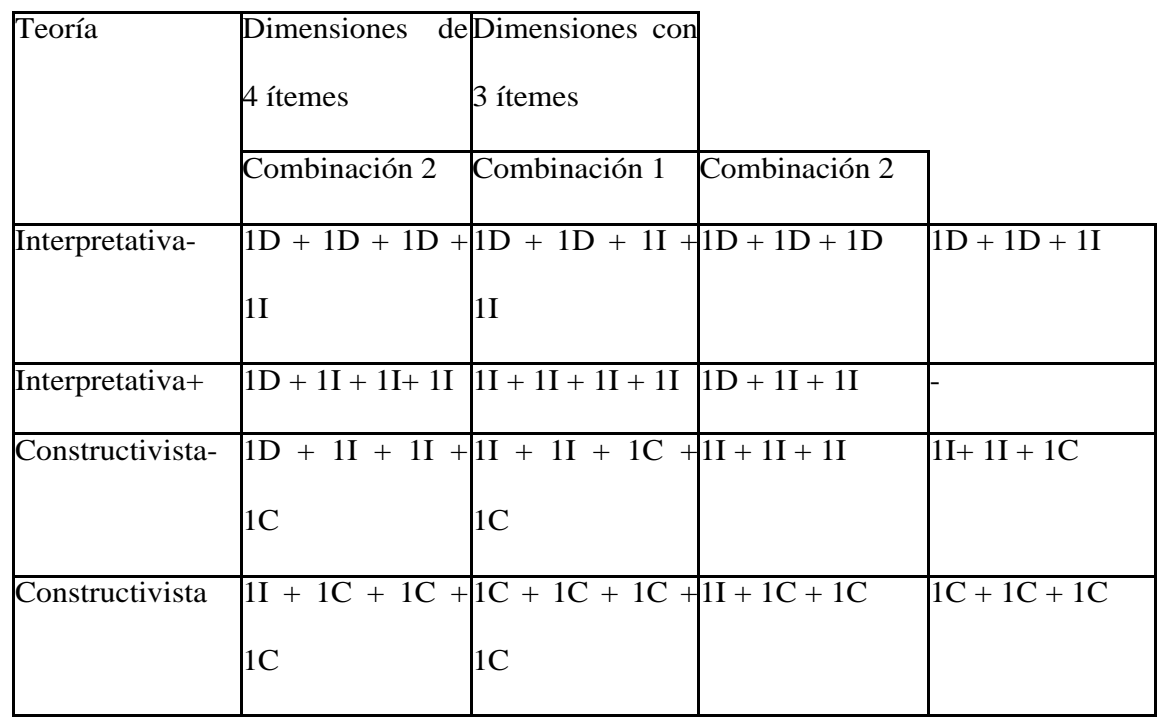

Tabla 10. Criterios para determinar el enfoque teórico en las distintas dimensiones evaluadas por el cuestionario.

Nos parece interesante reconocer las semejanzas y diferencias entre los grupos en cada dimensión de la enseñanza y el aprendizaje. Para realizamos análisis tanto descriptivos como comparativos utilizando la prueba de Chi-cuadrado y el programa SPSS versión 15.0

La tabla 11 muestra los porcentajes de profesores y estudiantes en cada tipo de enfoque, por dimensión. Como puede observarse, la categoría Constructivista- es la que parece hacer el peso en las diferencias entre los grupos, aunque en la dimensión enseñanza de actitudes aparece un porcentaje importante de Constructivista+ en ambos grupos.

\begin{tabular}{|c|c|c|c|c|c|c|c|c|}
\hline Dimensiones/sujetos & Profesores & Estudiantes & & & & & & \\
\hline 1- & $1+$ & C- & $C+$ & - & $1+$ & C- & $\mathrm{C}+$ & \\
\hline Conceptos* & 14.5 & 55.8 & 21.5 & 8.2 & .5 & 45.2 & 46.6 & 7.6 \\
\hline Procedimientos* & 12.4 & 40.4 & 37.1 & 10.0 & 1.7 & 24.1 & 56.2 & 17.8 \\
\hline Actitudes* & 1.2 & 9.6 & 52.6 & 36.7 & 2.4 & 14.1 & 36.7 & 46.6 \\
\hline Motivación* & .2 & 19.9 & 60.3 & 19.6 & 7.3 & 24.4 & 47.4 & 20.7 \\
\hline Evaluación* & 1.9 & 32.9 & 58.9 & 6.3 & 3.9 & 41.7 & 47.9 & 6.3 \\
\hline Habilidades/contenidos & 6.3 & 37.4 & 38.8 & 17.5 & 6.6 & 36.9 & 32.2 & 24.1 \\
\hline
\end{tabular}

Tabla 11. Porcentajes de cada perfil respecto a las 6 dimensiones evaluadas, en profesores y estudiantes 
Los análisis muestran que existen diferencias significativas entre ambos grupos. Los estudiantes son más constructivistas que los profesores en las dimensiones de enseñanza de conceptos $(\mathrm{X} 2(3, \mathrm{~N}=838)=97.393, \mathrm{p}<.000)$ y enseñanza de procedimientos. $(\mathrm{X} 2$ (3, $\mathrm{N}=838)=76.098, \mathrm{p}<.000)$. Es decir, los estudiantes enfatizarían más el carácter "dialógico" en la enseñanza, utilizando interacciones profesor-alumnos para generar espacios de contrastación conocimientos previos-contenidos disciplinares que permitan comprender, además, cómo el conocimiento se aplica a distintos contextos y es visto como necesario o significativo por los alumnos, en contraste con la tendencia de los profesores hacia metas reproductivas basadas en la presentación del conocimiento "correcto" que debe ser asimilado por los estudiantes. Las diferencias se vuelven a favor de los profesores en servicio en las dimensiones de motivación $(\mathrm{X} 2(3, \mathrm{~N}=838)=36.743, \mathrm{p}<.000)$, evaluación $(\mathrm{X} 2(3, \mathrm{~N}=838)=11.926, \mathrm{p}<.008)$ y enseñanza de actitudes $(\mathrm{X} 2(3, \mathrm{~N}=838)=22.095$, $\mathrm{p}<.000)$, quienes muestran en estos casos un enfoque más cercano al constructivismo que los estudiantes de pedagogía. Esto quiere decir que los profesores estarían más a favor del desarrollo de la autonomía y la reflexión por parte del alumno y los estudiantes de pedagogía se inclinan más hacia el rigor y el control del ambiente respecto de estas dimensiones. No se encuentran diferencias significativas entre profesores y estudiantes respecto a la dimensión habilidades versus contenidos.

\section{DISCUSIÓN Y CONCLUSIONES}

El estándar setting nos permitió conformar distintos perfiles teóricos puesto que los sujetos utilizaron los tres enfoques teóricos para responder a las situaciones presentadas en el cuestionario. Diferentes estudios muestran cierta co-existencia teórica, similar a perfiles o tipologías (Galaz 2007; Martín en evaluación; Mortimer 2001; Tillema 1997) más que enfoques puros, pero el problema es cómo dar cuenta de esta mixtura. El equipo de Martín (en evaluación), en un estudio con 1716 profesores en formación con 20 distintas áreas de especialización, realizó análisis de Clusters. De este modo, conformó tres grupos de sujetos, a saber, interdirectos, interconstructivos y constructivos. Sus resultados tienen cierta similitud con los nuestros ya que el grupo predominante es el que presenta una combinación de respuestas de tipo procesamiento de la información, constructivista, y directa (48.7\% de sujetos). En esta investigación hemos optado por definir criterios de corte en función de los porcentajes de las teorías y así hemos obtenido criterios más exigentes a la hora de clasificar a un sujeto como predominantemente constructivista, puesto que consideramos la contradicción epistemológica que significa la combinación de la visión constructivista con la del procesamiento de la información. Martín encuentra un $38.5 \%$ de los sujetos con un perfil constructivo versus el $10 \%$ de nuestro estudio. Estos resultados nos permiten afirmar que no es extraño entonces que los profesores muestren una enseñanza catalogada como "en transición", puesto que con este perfil teórico tendrán la misma probabilidad de interpretar situaciones desde el enfoque tradicional que desde un enfoque constructivista, sin que exista mayor consciencia sobre su existencia.

Si bien los estudiantes presentan mayor porcentaje de sujetos con un perfil constructivista+, este resultado muestra un modesto impacto de los programas de formación, manteniéndose la tendencia mostrada por los estudios de los años '90. Sin embargo, aunque no lleguen en su conjunto a una visión constructivista del aprendizaje y la enseñanza, 
podríamos considerar que los grupos interpretativo+ y constructivista- poseen una afinidad mínima suficiente como para intentar tirar desde ellas y producir el cambio conceptual deseado. Hay una gran diferencia entre considerar a los alumnos como una tábula rasa y considerarlos como poseedores de una mente con unos procesos cognitivos potentes para acercarse al conocimiento. El salto epistemológico aprovechando estas condiciones podría hacerse más fácil en los estudiantes de pedagogía, pero sería necesaria una intencionalidad mayor por parte de los formadores de profesores (Hyslop-Margison y Strobel, 2008; Windschitl, 2002). Nuestros resultados nos muestran que algunas universidades forman a estudiantes con teorías eminentemente interpretativas y otras más constructivistas, pero al comparar a los estudiantes de pedagogía pertenecientes a las instituciones que participaron en el proyecto de fortalecimiento a la formación inicial docente (PFFID), nuestros datos indican que no hay diferencias significativas entre los programas que participaron en esta convocatoria y quienes no lo hicieron. Esto nos indica que es necesario realizar un análisis crítico de las barreras, resistencias o dificultades que podrían haberse presentado en la implementación de tales propuestas, especialmente cuando la orientación de la formación de profesores ha tenido realmente un enfoque constructivista.

Hoban (2003) señala que los programas de formación inicial mantienen una estructura mecanicista al organizar el currículum de forma compartimentada a través de cursos independientes. Además, los formadores de profesores continúan realizando prácticas pedagógicas obsoletas (OCDE 2008). Gajardo (2009) ha encontrado, en el contexto de su tesis doctoral, que en tres de las instituciones formadoras de profesores más importantes de la ciudad de Santiago de Chile, el porcentaje de formadores de profesores que sostiene una visión constructivista del aprendizaje no supera el $20 \%$ del cuerpo académico. Según Lunenberg \& Korthagen (2003), para avanzar es necesario "romper el círculo" e introducir nuevas prácticas educativas en la formación de profesores.

Por otra parte, aún cuando éstos posean una visión constructuvista del aprendizaje, eso no asegura el cambio en las prácticas de enseñanza ni en el formador ni en sus estudiantes de pedagogía; los formadores de profesores rara vez usan el modelamiento ante sus alumnos en una forma productiva como para hacer explícita su propia enseñanza, y la conexión entre ésta y la teoría, no se "desarrollan [sic] automáticamente con los años: la experiencia como formador de profesores no necesariamente lleva a más y mejor modelamiento" (Lunenberg, Korthagen y Swennen, 2007: 597). Los autores sugieren realizar no sólo auto-estudios sino también observaciones y discusiones de las prácticas para promover el modelamiento, especialmente si esto se hace de forma estructurada.

Wideen et al. (1998), en una revisión de 93 estudios relacionados con el tema, encuentran que la recomendación más común era que el paso esencial del proceso consistiría en examinar los conocimientos previos de los futuros profesores. Nuestro desafío es avanzar hacia el desarrollo de más profesores constructivistas para nuestro sistema educativo, pero debemos evitar poner demasiada atención en las creencias, actitudes y conocimiento de los estudiantes -lo que a menudo ha llevado a que éstos comprendan los principios ligados a su quehacer pedagógico- sin entregarles suficientes herramientas prácticas para concretarlos en su enseñanza (McDonald 2005). Según Hyslop-Margison y Strobel (2008), los formadores de profesores deberían diseñar actividades de naturaleza transformadora, que promuevan el diálogo inter e intrapersonal acerca de los conceptos e ideas introducidas en el aula, enganchando a los estudiantes en la resolución de problemas complejos y poco estructurados dentro de contextos auténticos y aplicando conceptos y 
principios a situaciones nuevas y desafiantes. Esta y otras estrategias deberían ponerse a prueba tanto en la formación inicial y continua de los profesores e informar sobre sus resultados de investigación a la comunidad más amplia.

Otro resultado interesante es que los profesores con menos experiencia docente -hasta 6 años de servicio- poseen mayor predominio de enfoques constructivistas por sobre los profesores con más antigüedad. Este resultado coincide con el estudio de Martín (en prensa), en el que se observa un efecto positivo que se extendería hasta los diez primeros años de experiencia profesional. Nuestros datos indican que los docentes con menor experiencia docente no se diferencian de los profesores en formación, es decir, presentan enfoques teóricos similares. Esto podría estar indicando que existiría una leve tendencia a conservar intactos los enfoques promovidos en la universidad, dentro del grupo de profesores más jóvenes. Sin embargo, este hecho podría interpretarse de formas diferentes. Primero, se podría pensar que está disminuyendo el efecto negativo del contexto escolar, cuya práctica habitual no suele responder a una visión constructivista del aprendizaje. Pero también podría ser efecto de la influencia de un pensamiento o discurso generacional compartido, sin que eso signifique una práctica de enseñanza coherente con dicho discurso. Por esta razón, estos resultados deben ser tomados con precaución. Se sugiere realizar estudios longitudinales que permitan hacer un seguimiento de este aspecto o bien refinar más aún la forma en que se modifican eficientemente las teorías implícitas sobre la enseñanza y el aprendizaje y la forma en que este cambio se conecta con un cambio en las prácticas de enseñanza de los futuros profesores. Una tercera posibilidad podría relacionarse con lo que señala Klein (1997) respecto a que en la formación existe un constante interjuego entre sujetos, conocimiento y poder/autoridad, el que determina qué formas son más promisorias para posicionarse positivamente dentro del discurso (los estudiantes desean obtener buenas notas y el académico el reconocimiento de sus pares y estudiantes). Según esta autora, los estudiantes aprenden a regular el discurso y esta habla es evaluada de acuerdo a cuánto se ajusta con el marco entregado por el profesor. Por lo tanto, este resultado podría prevenirnos de la posibilidad de estar preparándolos para reconocer el discurso constructivista más que impactando en los aspectos nucleares que permiten el cambio conceptual.

Respecto a los docentes en ejercicio, merece ser reflexionado y profundizado el bajo impacto que tiene la formación continua en las concepciones del aprendizaje y la enseñanza. Esto es especialmente importante cuando consideramos que, para asumir la reforma educacional, estos programas debieran orientar a los profesores con nuevas teorías y estrategias metodológicas coherentes con dichos enfoques. Una crítica a los mecanismos de formación permanente la encontramos en Cohen \& Hill (2000, citados en Ball et al., 2001) quienes señalan que ésta generalmente consiste en talleres intelectualmente superficiales, desconectados de temas profundos del currículo y del aprendizaje, además de fragmentados y no acumulativos. Para generar este tipo de cambio no basta con instancias de desarrollo profesional aisladas de la práctica docente sino que debiéramos integrarla a lo que ocurre en las escuelas, como postulan los enfoques de reflexión sobre la práctica. Sin embargo, esto pone de relieve otro punto importante: la necesidad de generar cambios estructurales en las condiciones de trabajo de los profesores, que les permita generar la reflexión necesaria para modificar sus concepciones de aprendizaje y, por tanto, sus propias prácticas de enseñanza. No debería sorprendernos que los profesores de colegios particulares pagados tengan visiones teóricas más cercanas al 
constructivismo, puesto que en este tipo de escuelas se cuenta con mayor proporción de horas no destinadas directamente a la enseñanza y que, por tanto, pudieran ser utilizadas para la reflexión sobre la enseñanza y el aprendizaje. En cambio, los profesores pertenecientes a colegios particulares subvencionados -los que presentan condiciones laborales más precarias- tienen los porcentajes más altos de horas dedicadas directamente a la docencia. Esto podría estar incidiendo en la mantención de enfoques del aprendizaje más tradicionales. Según Liston, Borko y Whitcomb (2008), la asignación apropiada de tareas; la calidad de la inducción y la mentoría; la alineación del currículum con la escuela; la calidad del desarrollo profesional continuo y la cultura de aprendizaje profesional entre los profesores; la adecuación de las facilidades y recursos y la calidad del liderazgo disponible, son factores que importan a la hora de asegurar la calidad de los profesores.

Respecto a las diferencias encontradas entre profesores en servicio y estudiantes respecto a las distintas dimensiones de la enseñanza y el aprendizaje, los estudiantes de pedagogía tendrían una visión más constructivista respecto a la enseñanza de conocimientos declarativos y procedimentales, no obstante, a la hora de evaluar, motivar y enseñar actitudes sus posturas son bastante reproductivas. Comprenden correctamente que se necesita un cambio en las actividades de enseñanza, pero aún no logran consistencia con las otras dimensiones de ésta. Por su parte los profesores no han cambiado sus prácticas de reproducción del conocimiento, pero consideran relevante la autonomía del alumno. Éste es un problema puesto que no puede haber autonomía si ésta no es mediada adecuadamente por los profesores. Como se evidencia en este estudio, todavía tenemos mucho que aprender sobre cómo desarrollar enfoques sobre la enseñanza y el aprendizaje más acordes con los cambios del siglo XXI. Esto nos desafía no sólo a observar lo que ocurre en los profesores en formación o en servicio sino también a revisar profundamente los programas de formación y, en especial, la formación de los propios formadores de profesores.

\section{REFERENCIAS BIBLIOGRÁFICAS}

Avalos, B. (2004, septiembre). Las instituciones formadoras de docentes y las claves para formar buenos docentes. Ponencia presentada en $I^{\circ}$ Encuentro Internacional de la Red de Formación Docente de América Latina y El Caribe, El Desafío de Formar los Mejores Maestr@s. San Pedro Sula, Honduras.

Ball, D., Lubienski, S. \& Newborn, D. (2001). Research on teaching mathematics: The unsolved problem of teacher's mathematical thinking. En V. Richardson, Handbook of Research on Teaching (pp. 433-456). USA: American Educational Research Association.

Beca, C. Montt, P, Sotomayor, C., García - Huidobro, J. \& Walter, H. (2006). Docentes para el nuevo siglo. Santiago: Ministerio de Educación. Serie Bicentenario.

Brooks, J. G., y Brooks, M. G. (1993). The case of constructivist classrooms. ASCD: Alexandria, VI. Brown A. L. (1994) The advancement of learning. Educational Researcher, n. 23, 4-12.

Cox, C. (2003). Las políticas educacionales de Chile en las últimas dos décadas del siglo XX. En C. Cox (Ed.), Políticas Educacionales en el Cambio de Siglo. La reforma del sistema escolar en Chile (pp. 19-113). Santiago: Editorial Universitaria.

Dweck, C. (1996). Implicit theories as organizers of goals and behavior. En Peter Gollwittzar and John Bargh, The Psychology of Action: Linking cognition and motivation to behavior (pp. 69-90). New York, NY: The Guilford Press: 
Gajardo, M. (2008). Elicitando teorías implícitas sobre la enseñanza y el aprendizaje en formadores de profesores y estudiantes de Pedagogía en Educación General Básica. Tesis doctoral no publicada. Pontificia Universidad Católica de Chile, Santiago, Chile.

Galaz, A. (2007). Desarrollo profesional docente y (re) construcción de la identidad profesional de los profesores de enseñanza media: el caso de los grupos profesionales de trabajo. Tesis doctoral no publicada. Santiago: Pontificia Universidad Católica de Chile.

Hatano, G., \& Inagaki, K. (1994) Young children's naive theory of biology. Cognition, 50, 171-188.

Hoban, J (2003). The complexity if learning to teach: A four dimensional approach to designing teacher education program. Paper presentado al Annual Meeting of the American Educational Research Association in Chicago, IL. Abril, 2003.

Hyslop-Margison, E. \& Strobel, J. (2008) Constructivism and education: missunderstanding and pedagogical implications. The Teacher Educator, 43, 72-86.

Keil, F. C. (1992). The origin of an autonomous biology. En M. R. Gunnar, \& M. Maratsos (Eds.), Modularity and constraints in language and cognition. The Minnesota Symposia on Child Psychology (pp. 103-137). Erlbaum: Hillsdale.

Klein, M. (1997) Looking Again at the 'Supportive' Environment of Constructivist Pedagogy: an example from preservice teacher education in mathematics. Journal of Education for Teaching, vol. 23, n. 3, 277-292.

Korthagen, F., Loughran, J. \& Russell, T. (2006) Developing fundamental principles for teacher education programs and practices. Teaching and Teacher Education, 22, 1020-1041.

Liston, D., Borko, H. \& Whitcomb, J. (2008) The teacher educator's role in enhancing teacher quality. Journal of Teacher Education, vol. 59, n. 2, 111-116.

Lunenberg, M. \& Korthagen, F. (2003) Teachers educators and student direct learning. Teaching and Teacher Education 19: 29-44.

Lunenberg, M. Korthagen, F. \& Swennen, A. (2007). The teacher educator as a role model. Teaching and Teacher Education, n. 23, 586-601.

Martín, E. Variables personales y de centro asociadas con las concepciones de los docentes sobre el aprendizaje y la enseñanza. En prensa.

McDonald, M. (2005) The integration of social justice in teacher education: Dimensions of prospective teacher's opportunities to learn. Journal of Teacher Education, vol. 56, n. 5: 418-435.

Mortimer, E. (2001). Perfiles conceptuales: Modos de pensar y modos de hablar en las clases de ciencias. Infancia y Aprendizaje 24: 475-490.

OCDE (2008). Desafios de la educación superior en Chile. Extraído el 22 de diciembre de 2008 dehttp://www.universia.cl/html_estatico/portada/actualidad/noticia_actualidad/param/ noticia/ bdhahg.html

Pozo, J, I., Scheuer, N., Mateos, M. \& Pérez Echeverría, M. (2006). Las concepciones de los profesores de educación primaria sobre la enseñanza y el aprendizaje. En Pozo, J., Scheuer, N., Pérez Echeverría, M., Mateos, M, Martín, E \& de la Cruz, E., Nuevas formas de pensar la enseñanza y el aprendizaje (pp. 171-188). Graó: Barcelona.

Prawat, R. S. (1992) Teachers' beliefs about teaching and learning: A constructivist perspective. American Journal of Education, n. 100, 354-395.

Rodrigo, MJ. Y Correa, N. (2001). Representación y procesos cognitivos: esquemas y modelos mentales. En: C. Coll, J. Palacios y A. Marchesi, Desarrollo Psicológico y Educación, (T. 2, pp. 117-135). Alianza: Madrid.

Rodrigo, M.J., Rodríguez, A. y Marrero, J. (1993). Las teorías implícitas: Una aproximación al conocimiento cotidiano. Madrid: Visor.

Strauss, S. \& Shilony, T. (1994). Teacher's model of children's mind and learning. En L. Hirschfeld y S. Gelman (Eds.) Mapping the mind (pp. 275-299). Cambridge, MA: Cambridge University Press. 
Tillema, H. (1997). Stability and change in student teachers' beliefs. European Journal of Teacher Education, n. 20, 209-212.

Von Wright, M. (1997) Student Teachers' Beliefs and a Changing Teacher Role. European Journal of Teacher Education, n. 20, 257-266.

Wideen, M., Mayer-Smith, J. \& Moon, B. (1998) A critical analysis of the research on learning to teach: Making the case for an ecological perspective on inquiry. Review of Educational Research, vol. 68, n. 2, 130-131.

Windschitl, M. (2002) Framing Constructivism in Practice as the Negotiation of Dilemmas: An Analysis of the Conceptual, Pedagogical, Cultural, and Political Challenges Facing Teachers. Review of Educational Research, n.72, 131-175.

Woolfolk Hoy, A., Davis, H., \& Pape, S. (2006). Teachers' knowledge, beliefs, and thinking. En P. A. Alexander \& P. H, Winne (Eds.), Handbook of Educational Psychology (pp. 715-737). Mahwah, NJ: Lawrence Erlbaum. 
